\title{
Development and psychometric properties of the physical rehabilitation services acceptability questionnaire
}

\author{
Marzieh Shirazikhah ${ }^{1}$, Arash Mirabzadeh ${ }^{2}$, Homeira Sajjadi ${ }^{3}$, Mohammad Taghi Joghataei ${ }^{4}$, \\ Akbar Biglarian ${ }^{5}$, Farahnaz Mohammadi Shahboulaghi ${ }^{* 6}$, Hamid Sharif Nia ${ }^{7}$, Kelly A Allen ${ }^{8}$
}

\section{Abstract \\ Background: Access to rehabilitation services is considered to be a right for all people.} One of the most important indicators for access to rehabilitation services is an individual's general acceptance of rehabilitation. This tool was designed based on relevant studies and experiences of rehabilitation specialists to design a questionnaire to specifically measure patient acceptability of physical rehabilitation services.

Methods: In this study, an exploratory sequential mixed methods design was used. The first phase included a review of the literature and analysis of relevant studies, focus group discussions, and qualitative content analysis. In the second phase, construct validity was assessed by exploratory and confirmatory factor analysis. Also, convergent and divergent validity were measured. Reliability was evaluated by internal consistency (Cronbach's alpha and McDonald's Omega) and construct reliability. Statistical procedures were calculated by SPSS-AMOS24 and JASP0.9.2 software.

Results: A total of 200 questionnaires were completed by members of Iranian Disability Campaign. Three factors and 25 items were identified according to results of the first phase of this study. In the second phase, face validity was confirmed. To assess the content validity ratio, 9 items, with the mean of content validity ratio $(\mathrm{CVR})<0.49$, were deleted, while the content validity index $(\mathrm{CVI})<0.79$ was revised. The kappa coefficient $<0.6$ was fair and scale content validity index (SCVI) under 0.9 was considered appropriate. Results of exploratory factor analysis showed that $48 \%$ of the variance of the acceptability of physical rehabilitation services was based on patients' satisfaction, ethical behavior, and patient centered services. Confirmatory factor analysis confirmed the suitability of the final model. Convergent and divergent validity and reliability of the measure, the Physical Rehabilitation Services Acceptability questionnaire was fulfilled.

Conclusion: Findings indicated that the proposed constructs that promoted the Acceptability of Physical Rehabilitation Services Questionnaire had good validity and reliability in participants with physical disabilities.

Keywords: Psychometric properties, Acceptability, Physical rehabilitation

Conflicts of Interest: None declared

Funding: None

\section{*This work has been published under CC BY-NC-SA 1.0 license.}

Copyright $\odot$ Iran University of Medical Sciences

Cite this article as: Shirazikhah M, Mirabzadeh A, Sajadi H, Joghataei MT, Biglarian A, Mohammadi Shahboulaghi F, Sharif Nia H, Allen KA. Development and psychometric properties of the physical rehabilitation services acceptability questionnaire. Med J Islam Repub Iran. 2019 (24 Apr);33:34. https://doi.org/10.47176/mjiri.33.34

\section{Introduction}

The process of rehabilitation describes the return to

optimal functioning following the experience of disabil-

Corresponding author: Dr Farahnaz Mohammadi Shahboulaghi, mohammadifarahnaz@gmail.com

1. Social Determinants of Health Research Center, University of Social Welfare and Rehabilitation Sciences, Tehran, Iran

2. Social Determinants of Health Research Center, Psychiatry Department, University of Social Welfare and Rehabilitation Sciences, Tehran, Iran

3. Social Welfare Management Research Center, University of Social Welfare and Rehabilitation Sciences, Tehran, Iran

4. Cellular and Molecular Research Center, Department of Anatomy and Neuroscience, School of Medicine, Iran Medical University, Tehran, Iran

5. Department of Biostatistics, Social Determinants of Health Research Center, University of Social Welfare and Rehabilitation Sciences, Tehran, Iran

6. Iranian Research Center on Aging, Nursing Department, University of Social Welfare and Rehabilitation Sciences, Tehran, Iran

7. Amol Faculty of Nursing and Midwifery, Mazandaran University of Medical Sciences, Sari, Iran

8. Melbourne Graduate School of Education, University of Melbourne, Melbourne, Australia $\uparrow$ What is "already known" in this topic:

Access to rehabilitation services is important for people with disability. One of the most important indicators for accessing rehabilitation services is an individual's general acceptance of rehabilitation. In this study, the psychometric properties of the Physical Rehabilitation Services Acceptability Questionnaire were assessed.

$\rightarrow$ What this article adds:

This questionnaire allows policymakers, executive directors, and therapists to tailor their programs. The findings have implications for revising rehabilitation services that are currently provided in Iran and for developing new services in accordance with the unique needs of this group in the community. 
ity, illness, or injury (1). "Disability is the umbrella term for impairments, activity limitations, and participation restrictions" (3). World Health Organization, by promoting universal health coverage, advocates that all individuals should have access to health promotion, prevention, treatment, rehabilitation, and palliative services, based on their unique needs and irrespective of their geographical location or socioeconomic status $(2,3)$. Difficulties in accessing rehabilitation services can increase the negative impact of the disability, injury, or illness and delay rehabilitation for some individuals. Some individuals may be unable to participate in daily activities, and therefore their quality of life is diminished (3).

A growing elderly population and an increase in chronic illness have contributed to the rising incidence of individuals classified as having a disability, that is, impairment in body function or structure (4). It is estimated that one billion individuals (or $15 \%$ of the world's population) have a disability of some degree. Based on these estimates, in Iran, it is anticipated that more than 11 million people have disabilities and are in need of rehabilitation services $(5,32,33)$.Analysis conducted by the World Health Organization indicates that people with disabilities are 2 times more likely to encounter problems in accessing health care and rehabilitation services compared to healthy individuals (6). Access to rehabilitation services is considered to be a right for all people (7).

One of the most important indicators for accessing rehabilitation services is an individual's general acceptance of rehabilitation. Acceptability of rehabilitation services means that the individual perceives that rehabilitation services are accessible and provided with consideration of cultural, religious, physical, psychological, gender, and social differences $(7,8,9)$. Studies done on tools that measure people's acceptance of health and rehabilitation services have used various methods and parameters. In Australia, in 2013, Van Gaans investigated the access to cardiac rehabilitation services and identified a number of relevant indicators of acceptability, such as referrals from individuals who had previously used the service, insurance coverage, the gender of the care provider, and overall satisfaction with the service (10).

In 2010, another study assessed the recipient's cultural and religious beliefs, the degree of satisfaction with the service provider, the degree of satisfaction with the location of the service, and the degree of satisfaction with the professional skills of the service providers (11). In 2007, Obrist et al conducted a review to evaluate the access to primary health care services. In this study, 2 factors were determined to measure acceptability of service: satisfaction with the skill of the service provider and overall satisfaction with the services (12).

Overall, despite efforts of past research, very few comprehensive questionnaires exist for assessing the acceptability of rehabilitation services for people with disabilities. Creating a local questionnaire to measure the acceptability of rehabilitation services could be an appropriate tool to determine cultural barriers to access such services. This questionnaire can help the policymakers, service providers, and future researchers to evaluate the services and to design the services required based on the acceptance of the target community (3). In the world and in Iran, no questionnaire is available to measure the acceptability of rehabilitation services; therefore, the present study aimed to address this gap in the empirical literature.

\section{Methods}

An exploratory sequential mixed methods design was selected as the best fit for the purpose of this study. The design consisted of a primary quantitative data collection and analysis, followed by qualitative data collection and analysis (13).

\section{Overview of the research phases \\ 1. Phase 1: Qualitative \\ 1-1 Review of the literature}

An extensive literature review was done to investigate the acceptability of health services and rehabilitation. Keyword searches "Health Service", "Healthcare", "services health care", and "Rehabilitation and Acceptability" were used in different databases (eg, Google Scholar, Science Direct, PubMed, Magiran, SID, and IranMedex). All retrieved articles were reviewed. Inclusion criteria were as follow: (1) articles and documents related to the subject which were in accordance with the definitions provided regarding the acceptability of the service; (2) articles published between 2000 and 2017.

A preliminary search yielded 256 articles, but after removing duplicate articles, 174 articles were selected. Abstracts of these articles were evaluated and 81 articles were excluded, as they did not meet the inclusion criteria. The quality of articles was checked by 2 members of the research team and those not methodologically appropriate were excluded. A total of 5 articles that were in line with the objectives of the study were selected and analyzed. Selected literatures were analyzed by thematic analysis method.

\section{1-2. Focus groups}

To obtain the experts' opinion, the results of the assessment done on the selected articles were made available to the specialists (Appendix 1) through 6 focus group discussion sessions (FGD). The focus group included 3 rehabilitation specialists, 1 psychiatrist, 1 social worker, 1 statistician, and 2 specialists who also had a disability, as a representative of the target community. During these sessions, factors and items related to the acceptability of physical rehabilitation services were identified. An informed consent was obtained from each participant, and they consented to recording the sessions. Audiotaped discussions were listened and written on paper. The researchers studied the documentation carefully and then analyzed them using content analysis method. Group discussions and data collections were continued until data saturation. Data coding and analysis in each meeting was done based on the categories obtained in the previous session.

\section{Phase 2 \\ 2-1. Face validity}

The qualitative method was used to evaluate the validity 
of the questionnaire. To determine face validity, 30 individuals with physical disabilities who were members of Iran Disability Campaign were interviewed face to face. Then, relevancy, ambiguity, and inadequacies and difficulty of understanding the concepts and words were examined.

\section{2-2. Content validity}

To determine content validity ratio (CVR), 15 specialists were selected to answer the 3-option Likert Scale questionnaire ( 1 = necessary; 2 = useful but not necessary; 3 = unnecessary). To assess content validity index (CVI), a specialist responded to all these 3 criteria: (1) clarification, (2) relevancy, and (3) simplicity. The scale content validity index (S-CVI/Ave was computed based on average scores of the content indices. Beck and Polit decided on a score of $\geq 0.9$ as an admissible S-CVI/Ave (14).

\section{2-3. Construct validity}

Construct validity was measured using maximum likelihood exploratory factor analysis (MLEFA). The Bartlett's test of sphericity and Kaiser-Meyer-Olkin (KMO) were used to assess the properness of the sample to conduct the factor analysis. Factor extraction was based on (i) eigenvalues $>1$; (ii) communalities $>.3$, and (iii) scree plots (15) by SPSS 24 software. The presence of a single item in the factor was estimated to be approximately 0.3 based on the following formula: $\mathrm{CV}=5.152 \div \sqrt{ } \mathrm{n}-2(16,17)$. In accordance to the 3-indicator rule, there should be at least 3 observed variables (items) and a factor must exist for every latent variable (18). The results obtained from MLEFA were verified by confirmatory factor analysis (CFA) and by examining the model fit indexes such as Chi-square $\left(\chi^{2}\right)$ test, Chi-square/degree of freedom ratio (normalized chi-square CMIN/DF), adjusted goodness-offit index $(\mathrm{AGFI})>.80$, parsimonious comparative fit in$\operatorname{dex}(\mathrm{PCFI})>.90$, normed fit index $(\mathrm{NFI})$, normed fit index (NFI), Tucker-Lewis index (TLI), relative fit index $(\mathrm{RFI})>.90$, root mean square error of approximation (RMSEA) .05 - .10 moderate, and standardized root mean square residual $($ SRMR $)<.09(19)$.

\section{Setting samples}

The study population composed of disabled individuals selected from Iran Disability Campaign. The campaign had 2500 people with disabilities from all Iran's provinces; of them, 500 were from Tehran. According to the inclusion criteria, 250 people had physical disabilities and 200 were willing to participate in the study. An acceptable sample size is 5-10 for each questionnaire (25). All 200 questionnaires were distributed among all people with disabilities. Questionnaires were collected after being completed. Inclusion criteria were as follow: (1) people with physical disability whose disability has occurred at least a year ago; (2) willingness to participate in the study. Individuals with another kind of physical disability, those not willing to participate in the study, or those who did not complete the questionnaire were excluded from the study.

2-4. Convergent \& divergent validity

The Fornell-Larcker criterion was used to assess convergent \& divergent validity. According to this criterion, average variance extracted (AVE), maximum shared squared variance (MSV), and average shared square variance (ASV) were estimated to assess the convergent and discriminant validity of the extracted factors of Physical Rehabilitation Services Acceptability Questionnaire. To establish convergent validity, (i) AVE should be greater than .5 and (ii) construct reliability (CR) should be greater than AVE. To meet the discriminant validity criteria, both MSV and ASV of each construct should be less than its AVE (20).

\section{2-5. Univariate and multivariate normality and outliers}

To evaluate normality, it is helpful to assess both univariate and multivariate normality. Univariate distributions were examined for outliers and skewness $(< \pm 3)$ and kurtosis $(< \pm 8)(21)$. Multivariate distributions were evaluated for normality using Mardia's coefficient of multivariate kurtosis, whereas a Mardia's coefficient $>8$ indicates deviation of multivariate normality (22). Multivariate outliers can be evaluated by evaluation of Mahalanobis distance $(\mathrm{p}<.0001)(23)$. Next, the missing data were evaluated by imputation pattern criterion.

2-6. Reliability

To evaluate the internal consistency of the questionnaire, coefficients of Cronbach's alpha $(\alpha)$ and McDonald's Omega $(\Omega)$ were estimated, and values greater than 0.7 were considered acceptable (24). Also, construct reliability (CR) was estimated.

\section{Ethical Consideration}

The study protocol was approved by the Ethics Committee of the University of Social Welfare and Rehabilitation Sciences (code: 1393227).

\section{Results \\ 1. Phase1: \\ Qualitative}

Based on the qualitative phase, 9 categories and 39 subcategories were extracted from the analysis of the related articles. Finally, after focus group discussions, 34 items in 5 factors were developed with the help of the experts and using qualitative content analysis.

\section{Phase 2 \\ First draft of the questionnaire}

A pool of 34 items were generated at the end of the first phase of the study. The research team evaluated and reviewed the items in 4 phases. After omitting repeated items, a 34-item questionnaire entered the psychometric evaluation phase.

\section{Face and content validity}

In face validity, no items were omitted and only 2 words were corrected. In content validity ratio, 9 items, with $(\mathrm{CVR})<0.49$, were deleted, and content validity index $(\mathrm{CVI})<0.79$ was revised. The kappa coefficient $<0.6$ was fair and SCVI under 0.9 was considered appropriate. Finally, 25 items entered the construct validity stage.

\section{Construct validity}

Among the total participants $(\mathrm{n}=200), 117$ were female (58.5\%), 119 (59.5\%) single, 123 (61.5\%) severely handi- 


\begin{tabular}{lclc}
\multicolumn{3}{l}{ Table 1. Demographical characteristics of the study participants (n=200) } \\
\hline Variables & No (\%) & Variables & No (\%) \\
\hline Age (Years) & & Employment & \\
$0-18$ & $13(6.5)$ & Employed & $55(22)$ \\
$19-59$ & $174(87)$ & Student & $15(7.5)$ \\
$>60$ & $13(6.5)$ & Housewife & $40(20)$ \\
Gender & & Retired & $12(6)$ \\
Female & $117(58.5)$ & Unemployed & $88(44)$ \\
Male & $83(41.5)$ & Social class & \\
Levels of disability & & Lower class & $6(3)$ \\
Mild & $20(10)$ & Lower middle class & $97(48.5)$ \\
Moderate & $57(28.5)$ & Middle class & $79(39.5)$ \\
Severe & $123(61.5)$ & Upper middle class & $9(4.5)$ \\
Marital status & & Upper class & $9(4.5)$ \\
Single & $119(59.5$ & Educational Status & \\
Married & $68(34)$ & Illiterate & $9(4.5)$ \\
Widow & $2(1)$ & Elementary School & $21(10.5)$ \\
Separated & $5(2.5)$ & High school & $94(47)$ \\
Divorced & $6(2)$ & Diploma & $74(37)$ \\
& & University & $2(1)$ \\
\hline
\end{tabular}

capped, and $94(47 \%)$ were in middle school. Also, 54 $(27 \%)$ were unemployed, but despite their disability, they were able to work. Of the participants, 97 (48.5\%) considered themselves to belong to the middle- and lower-social class (Table 1).

KMO and Bartlett's test were .844, and 2067.250, respectively $(\mathrm{p}<.001)$. In the MLEFA, results of the study indicated that the measure with 22 items comprised of client satisfaction, ethical behavior, and patient-centered services. Taken together, these factors were able to explain $48 \%$ of the total variance towards the acceptability of Physical Rehabilitation Services Questionnaire, which was extracted from the following factors: customers' satisfaction, ethical behavior, and patient-centered services. The Eigenvalue of these 3 latent factors were 5.43, 2.9, and 2.15 , respectively. As a whole, $48 \%$ of the total variance determined the acceptability of physical rehabilitation services (Table 2).

Table 2. Factor analysis for the acceptability of rehabilitation services questionnaire

\begin{tabular}{|c|c|c|c|c|c|}
\hline Factor & Items & Loading & $\begin{array}{c}\mathrm{h}^{2} \\
\text { (Communalities) }\end{array}$ & $\%$ of Variance & Eigenvalue \\
\hline \multirow{12}{*}{ 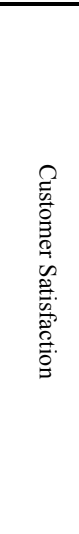 } & $\begin{array}{l}\text { Q 12. To what degree would you rate your satisfaction to the explanations given by the health care } \\
\text { provider while doing his rehabilitation services? }\end{array}$ & 0.802 & 0.669 & 24.667 & \multirow[t]{12}{*}{5.427} \\
\hline & $\begin{array}{l}\text { Q14.In your opinion, how would you rate you're your satisfaction with the type of relationships } \\
\text { provided by your rehabilitation service provider? }\end{array}$ & 0.800 & 0.658 & & \\
\hline & $\begin{array}{l}\text { Q11. How well did your service provider spend his time in listening to your problems and other issues } \\
\text { while rendering his services? }\end{array}$ & 0.793 & 0.631 & & \\
\hline & $\begin{array}{l}\text { Q15. How would you rate the professional skills and competencies of you rehabilitation service } \\
\text { provider? }\end{array}$ & 0.733 & 0.582 & & \\
\hline & $\begin{array}{l}\text { Q13. How would you rate the attention given by your health care provider and the respect for your } \\
\text { privacy in rendering health services? }\end{array}$ & 0.706 & 0.507 & & \\
\hline & Q10. How respectful and attentive were the health service providers when rendering their services? & 0.628 & 0.416 & & \\
\hline & Q25. As a whole, how would you rate the services being rendered to you? & 0.608 & 0.372 & & \\
\hline & Q23. How would you rate your involvement in the planning of rehabilitation services? & 0.566 & 0.345 & & \\
\hline & Q9. How would you rate the services of the health provider in terms of gender? & 0.558 & 0.323 & & \\
\hline & $\begin{array}{l}\text { Q5. How would you rate your satisfaction in the physical environment of the health service facility in } \\
\text { terms of (enough space, enough chairs, waiting room and pleasant environment). }\end{array}$ & 0.550 & 0.304 & & \\
\hline & $\begin{array}{l}\text { Q6. How would you rate the environmental condition of the health service facility in terms of (light- } \\
\text { ing, noise, ventilation, cleanliness and suitable temperature)? }\end{array}$ & 0.549 & 0.311 & & \\
\hline & Q22. How would you rate the respect for your right in receiving rehabilitation services? & 0.482 & 0.259 & \multirow{7}{*}{13.200} & \\
\hline \multirow{6}{*}{ 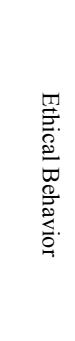 } & $\begin{array}{l}\text { Q17. How would you rate the use of harsh and unscrupulous methods of treatment as the reason for } \\
\text { your discontinuance of rehabilitation services? }\end{array}$ & 0.851 & 0.726 & & \multirow[t]{6}{*}{2.904} \\
\hline & $\begin{array}{l}\text { Q19. How would you rate inattention or neglect of the health service provider to your religious beliefs } \\
\text { as a reason for discontinuance of rehabilitation services? }\end{array}$ & 0.721 & 0.522 & & \\
\hline & $\begin{array}{l}\text { Q16.How would you rate carelessness and the lack of time spent by the health care provider as a } \\
\text { reason for discontinuance of your rehabilitation services? }\end{array}$ & 0.704 & 0.500 & & \\
\hline & $\begin{array}{l}\text { Q20. How would you rate the inattention of the health service provider to culture, customs and tradi- } \\
\text { tions and ethnicity as a reason for the discontinuance of your rehabilitation services? }\end{array}$ & 0.656 & 0.471 & & \\
\hline & $\begin{array}{l}\text { Q18. How would you rate disclosure of your information to others as the reason for your discontinu- } \\
\text { ance of your rehabilitation services? }\end{array}$ & 0.519 & 0.340 & & \\
\hline & $\begin{array}{l}\text { Q24. How would you rate the reason of your refusal to receive further rehabilitation services because } \\
\text { your health care provider believed that your condition would not improve? }\end{array}$ & 0.476 & 0.284 & & \\
\hline \multirow{4}{*}{ 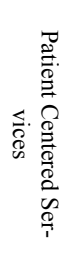 } & $\begin{array}{l}\text { Q3. How would you rate the lack of attention by health service provider to your condition as the } \\
\text { reason for discontinuing your health services? }\end{array}$ & 0.747 & 0.661 & \multirow[t]{4}{*}{9.770} & \multirow[t]{4}{*}{2.149} \\
\hline & $\begin{array}{l}\text { Q2. How would you rate your feeling that your physical and Psychological problems is of no im- } \\
\text { portance to the health care provider? }\end{array}$ & 0.727 & 0.557 & & \\
\hline & $\begin{array}{l}\text { Q1. How would you rate your experience of rejection from the Rehabilitation Services Center as the } \\
\text { reason for discontinuance of the rehabilitation services? }\end{array}$ & 0.628 & 0.539 & & \\
\hline & $\begin{array}{l}\text { Q7. How would you rate the absence of private room for examination and for performing rehabilita- } \\
\text { tion services as the reason for your discontinuance of the rehabilitation services? }\end{array}$ & 0.623 & 0.503 & & \\
\hline
\end{tabular}


In the CFA, the preliminary model did not show goodness-of-fit before modification (Table 3). After model modification and plotting the correlation between the measured errors, Chi-square goodness-of-fit test was done $\left(\mathrm{p}<.001, \chi^{2}=313.55,(\mathrm{~N}=250, \mathrm{df}=75)\right)$. Then, to assess the goodness-of-fit of the model, other indices were evaluated $(\mathrm{PCFI}=.784, \mathrm{PNFI}=.719, \mathrm{NFI}=.957, \mathrm{CFI}=.948$, $\mathrm{CMIN} / \mathrm{DF}=1.924, \mathrm{RMSEA}=.068, \mathrm{AGFI}=.831)$, which confirmed the goodness-of-fit of the final model (Table 3 and Fig. 1). As demonstrated in Table 4, AVE in all factors was $>.5$ and AVE for each factor was higher than ASV and MSV, respectively. Results showed that acceptability of physical rehabilitation services has an appropriate convergent and divergent validity. Internal consistency and CR was acceptable (>.7) (Table 4). Based on this re- sult, Physical Rehabilitation Services Acceptability Questionnaire was established (Appendix 2).

\section{Discussion}

The present study aimed to design a tool to measure the acceptability of physical rehabilitation services in patients with a disability. The psychometric features of the questionnaire were confirmed by the present results, confirming that this tool is a valid and reliable questionnaire to assess the acceptability of physical rehabilitation services among people with disability.

1. Phase 1: In this phase, according to the results of previous studies and focused group discussions, 5 factors, including feeling of stigma, physical location of service delivery, the skills and communication of service provid-

Table 3. Results of fit index confirmatory factor analysis of the questionnaire $(\mathrm{n}=200)$

\begin{tabular}{|c|c|c|}
\hline MEASURE & FIT INDEXES & VALUES \\
\hline P-value $\chi^{2}$ (Chi-squared P-value) & $>0.05$ & \\
\hline (Root Mean Square Error of Approximation) RMSEA & $>0.08$ good, 0.01 TO 0.08 moderate:,bad $<0.1$ & 0.068 \\
\hline (Comparative Fit Index) CFI & $>0.9$ & 0.948 \\
\hline (Normed Fit Index) NFI & $>0.9$ & 0.957 \\
\hline (Adjusted Goodness of Fit Index) AGFI & $>0.8$ & 0.841 \\
\hline (Minimum Discrepancy Function by Degrees of Freedom divided) CMIN/DF & 5>acceptable:, $3>$ good & 1.924 \\
\hline (Parsimonious Normed Fit Index) PNFI & $>0.5$ & 0.784 \\
\hline (Parsimonious Comparative Fit Index)PCFI & $>0.5$ & 0.784 \\
\hline
\end{tabular}

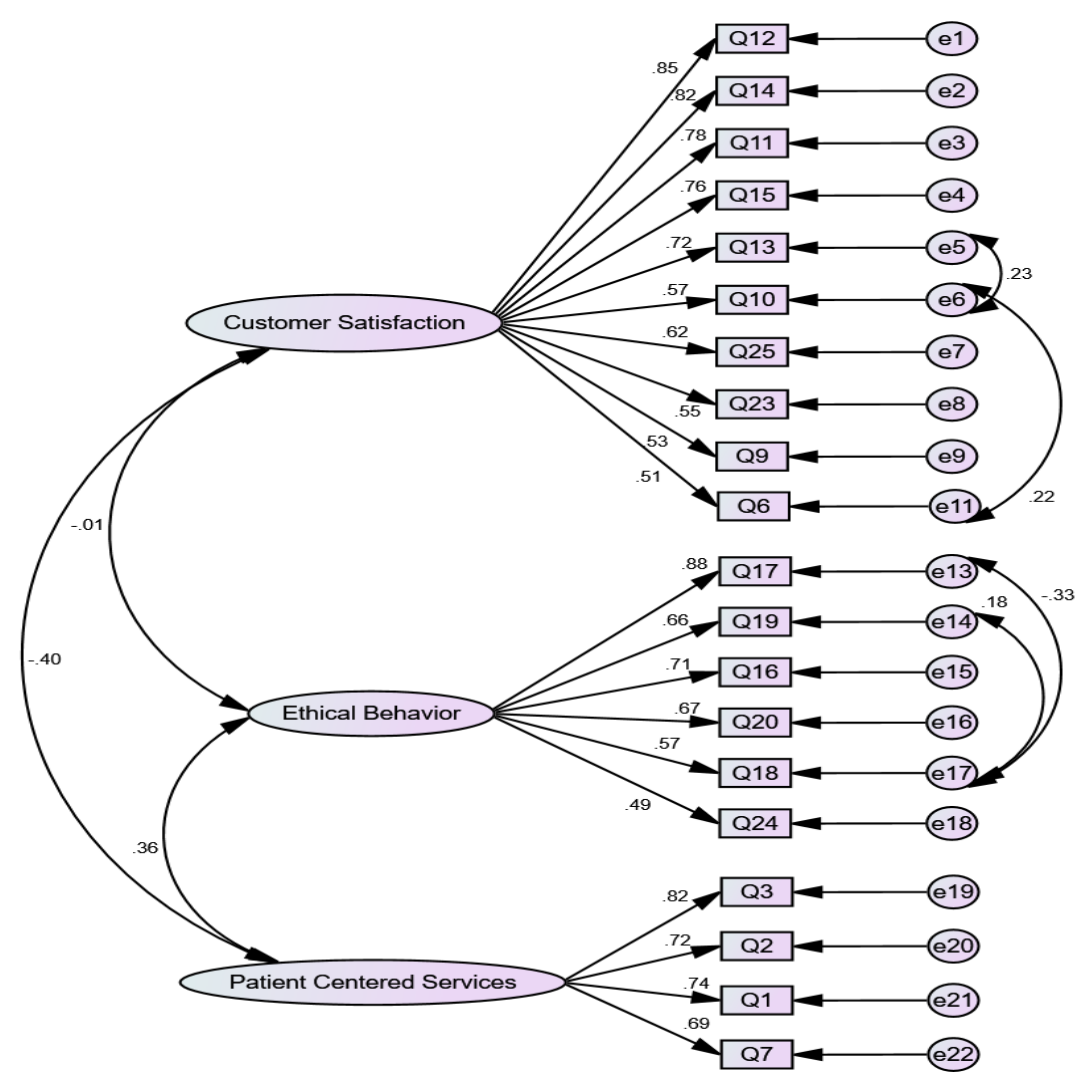

Fig. 1. The final model of the rehabilitation services acceptability model

Table 4. Convergent and divergent validity and reliability

\begin{tabular}{|c|c|c|c|c|c|c|}
\hline Factor & $\alpha$ & $\Omega$ & $\mathrm{CR}$ & AVE & MSV & ASV \\
\hline Customer Satisfaction & .897 & .708 & .900 & .568 & .158 & .079 \\
\hline Ethical Behavior & .823 & .729 & .829 & .555 & .127 & .064 \\
\hline patient centered services & .830 & .795 & .832 & .554 & .158 & .143 \\
\hline
\end{tabular}

Abbreviations. $\alpha$ : Cronbach's alpha coefficients, $\Omega$ : McDonald's Omega Coefficient, CR: Construct Reliability, AVE: Average Variance Extracted, MSV: Maximum Shared Squared Variance, ASV: Average Shared Squared Variance 
ers, kind of clients and personnel attitudes, individual communication skills, and overall satisfaction were identified.

In 2013, Van Gaans studied the items of measure of acceptability and introduced 2 items: (1) clients' attitudes about health workers and characteristics of existing heath providers, including age and sex; and (2) religion of the of provider or the provider's attitude about clients, including source of patient payment and ethnicity (10). In 2010, Shrestha conducted a study to evaluate the acceptability of primary health care and to measure satisfaction of sex, skill, cultural and religious background of the health worker, cleanliness of facility, and overall satisfaction (11).

2. Phase 2: In this phase, 3 factors were identified. The first factor in the questionnaire was client satisfaction. The concept of client satisfaction refers to whether or not the client (service recipient) is satisfied with the services and the service provider. Client satisfaction is an important determinant of whether the client will be accepting the rehabilitation service. In fact, the skills of the service provider, the quality of service, and factors related to the physical conditions of the service have been found to be essential for client satisfaction (26). For client satisfaction, the highest factor loading (0.8) was related to the item, "To what degree would you rate your satisfaction about the therapist's explanations while he/she is providing rehabilitation services"? and the lowest factor loading (0.48) was related to the item," To what degree would you rate your satisfaction with the therapist's respect of your right to choose the rehabilitation services you desire?" These results were consistent with those of other studies that focused on the skills of therapists $(11,12)$ and on their respect for patient autonomy (10).

Results of the exploratory factor analysis indicated that ethical behavior was the second dimension of acceptability of physical rehabilitation services. Ethical behavior is the adherence to ethical codes and conduct, promotion of an ethical climate and culture of an organization. Organizations should ensure that policies, programs, and practices are designed and presented ethically with attention to norms, religion, language, culture, and ethnicity (25). Ethical behavior had a variable factor loading (from 0.48 to 0.85 ), which was a finding consistent with the results of a previous study conducted by Shrestha, Pogge and VanGaans $(10,11,27)$.

The third factor extracted was the acceptability of patient-centered services in providing rehabilitation services. Important features of these services were as follow: (1) designing services based on the patient's physical, psychological, and social health needs; (2) providing services tailored to the facilities needed by the clients; and (3) eliminating social barriers, such as discrimination and social stigma (28). For this factor, the item that had the most significant loading was as follows: "To what degree would you rate the provider's lack of attention to your needs as your reason for the discontinuation of the rehabilitation services?" The item with the lowest loading was as follows: "To what degree would you rate the lack of private space for physical examination and receiving rehabil- itation services as your reason for discontinuation of your rehabilitation services?" VaanGaans suggests that the behavior of the service provider and the place of delivering services are important factors that determine a patient's acceptance of a service (10).

After the elimination of insignificant data and studying the data distribution, the functional structure of the physical Rehabilitation Services Acceptability Questionnaire was verified by confirmatory factor analysis. The most common indicators of goodness-of-fit were evaluated. Results of the study indicated that the goodness-of-fit model was suitable for all indicators.

In the present study, convergent and divergent validity showed that all factors had an appropriate convergent validity. Also, divergent validity of the factors was confirmed. Hear (1955) suggests that convergent validity can occur when constructs are close to each other and share a lot of variance. On the other hand, divergent validity can occur when constructs are actually unrelated (20). In a more literal sense, there is no suitable convergent validity when hidden variables are not well explained by the extracted constructs and the constructs are not sufficiently correlated to each other (29).

The stability of the Rehabilitation Services Acceptability Questionnaire was evaluated by Cronbach's alpha coefficients, Omega-McDonald's, and Theta, respectively. The Theta coefficient is one of the indicators of internal stability analysis when the principal components analysis is performed, and the Omega-Macdonald coefficient is estimated during exploratory factor analysis (30). Karmin and Zeller (1986) stated that among the 3 coefficients, the Cronbach's Alpha coefficient has the lowest scale score, while the Omega-McDonald's has the highest scale score (31).

\section{Limitations and strengths}

Limitations: One of the limitations of our research was the lack of access to the sample of the study. Because of the nature of the research, we could not take samples from the rehabilitation centers and we had to take the community. However, as we chose the samples from the campaign of people with disabilities and the number was limited, we had to run on the same sample as EFA and CFA. Nevertheless, and we used other tests to increase the validity of the study.

Strengths: In this study, using a qualitative and quantitative method, the acceptability of rehabilitation services questionnaire was developed. Moreover, The findings of this study suggest that this questionnaire can be helpful in evaluating the acceptability of physical rehabilitation services and can generate valuable evidence for developing new policies and improving access to these services.. In addition, this questionnaire can be a good model for designing future questionnaires on mental and psychological disabilities.

\section{Conclusion}

The present study aimed to design and validate an indigenous questionnaire for assessing the acceptability of physical rehabilitation services in Iran. The results indi- 
cated that the validity and reliability of the questionnaire were acceptable. The questionnaire allows the researchers to measure acceptability of physical rehabilitation services and provide the results to policymakers, executive directors, and therapists to tailor their programs.

\section{Conflict of Interests}

The authors declare that they have no competing interests.

\section{References}

1. Bethge M, von Groote P, Giustini A, Gutenbrunner C. The World Report on Disability: a challenge for rehabilitation medicine. Am J Phys Med Rehabil. 2014; 93:S4-11.

2. Evans DB, Hsu J, Boerma T. Universal health coverage and universal access. Bull World Health Org. 2013; 91:546-546A.

3. World report on disability 2011. Geneva: World Health Organization; 2011. p. 93-102.

4. World Health Organization. Disabilities. 2017. Available from: http://www.who.int/topics/disabilities/en/.

5. World Health Organization. Research for universal health coverage: World health report 2013. Available from: http://www.who.int/whr/2013/report/en/

6. World Health Organization. Disability and rehabilitation: WHO global disability action plan 2014-2021: Better health for all people with disability. 2015. p1-6. Available from: http://www.who.int/disabilities/actionplan/en/

7. World Health Organization. Human rights and health. 2017; Available from: http://www.who.int/mediacentre/factsheets/fs323/en/.

8. Skempes D, Bickenbach J. Strengthening rehabilitation for people with disabilities: a human rights approach as the essential next step to accelerating global progress. Am J Phys Med Rehabil. 2015; 94(10):823-828.

9. Tanahashi T. Health service coverage and its evaluation. Bull World Health Organ. 1978; 56:295-303.

10. van Gaans DA. The accessibility of Phase 2 Cardiac Rehabilitation Programs in rural and remote Australia. [Thesis]. South Australia: The University of Adelaide; 2013 p.34-44.

11. Shrestha J. Evaluation of Access to Primary Healthcare: A Case Study of Yogyakarta, Indonesia. University of Twente Faculty of Geo-Information and Earth Observation (ITC); 2010. p. 1-90.

12. Obrist B, Iteba N, Lengeler C, Makemba A, Mshana Ch, Nathan $R$, et al. Access to health care in contexts of livelihood insecurity: a framework for analysis and action. PLoS Med. 2007;4:1584-1588.

13. Creswell JW, Clark VLP. Designing and conducting mixed methods research. California: Sage Publications; 2007.

14. Polit DF, Beck CT. Essentials of nursing research: Methods, appraisal, and utilization. 6th ed. United States, Philadelphia: Lippincott Williams \& Wilkins; 2006.

15. Sharif Nia H, Pahlevan Sharif S, Koocher GP, Yaghoobzadeh A, Haghdoost AA, Mar Win MT, et al. Psychometric properties of the death anxiety scale-extended among patients with end-stage renal disease. Omega (Westport). 2017: 30222817733220.

16. Fok D. Development and testing of a low vision product selection instrument (LV-PSI): a mixed-methods approach. [Thesis]. The University of Western Ontario; 2011. p. 223.

17. Soleimani MA, Pahlevan Sharif S, Allen KA, Yaghoobzadeh A, Sharif Nia H. Psychometric properties of the Persian version of spiritual well-being scale in patients with acute myocardial infarction. J Relig health. 2017; 56:1981-1997.

18. Munro BH. Statistical methods for health care research. Lippincott Williams \& Wilkins; 2005.

19. Sharif Nia H, Pahlevan Sharif S, Lehto RH, Boyle C, Yaghoobzadeh A, Kaveh O, et al. Development and psychometric evaluation of a Persian version of the Death Depression Scale-Revised: a crosscultural adaptation for patients with advanced cancer. Jpn J Clin Oncol. 2017;47:713-719.

20. Hair J, Anderson RE, Tatham RL, Black WC. Multivariate Data Analysis with Readings, Pearson College Div; 1995. p. 178-237.

21. Sharif Nia H, Haghdoost AA, Ebadi A, Soleimani MA, Yaghoobzadeh A, Abbaszadeh A, et al. Psychometric properties of the king spiritual intelligence questionnaire (KSIQ) in physical veterans of Iran-Iraq warfare. Mil Med. 2015;17:145-153.

22. Raoprasert T, Islam SM. Designing an Efficient Management System: modeling of convergence factors exemplified by the case of Japanese businesses in Thailand. Springer Science \& Business Media; 2010.

23. Tabachnick BG, Fidell LS, Osterlind SJ. Using multivariate statistics. California State University; 2013.

24. Sharif Nia H, Pahlevan Sharif S, Boyle C, Yaghoobzadeh A, Tahmasbi B, Rassool GH, et al. The Factor Structure of the Spiritual Well-Being Scale in Veterans Experienced Chemical Weapon Exposure. J Relig Health. 2018 Apr;57(2):596-608.

25. Treviño LK, den Nieuwenboer NA, Kish-Gephart JJ. (Un) ethical behavior in organizations. Annu Rev Psychol. 2014;65:635-660.

26. Yadav MK, Rai AK. Exploring the relational impact of service quality on customer satisfication. Ushus-J Business Manag. 2015;14:17-31

27. Pogge T. Access to medicines. Public Health Ethics. 2008;1:73-82.

28. World Health Organization. People-centred services. 2017. Available from: http://www.who.int/csr/disease/ebola/health-systemsrecovery/services/en/.

29. Fornell C, Larcker DF. Evaluating structural equation models with unobservable variables and measurement error. J Mark Res. 1981. 3950

30. Ercan I, Yazici B, Sigirli D, Ediz B, Kan I. Examining Cronbach alpha, theta, omega reliability coefficients according to sample size. J Mod Appl Stat Methods. 2007; 6: 27

31. Carmines EG, Zeller RA. Reliability and validity assessment. Sage Publications; 1979 .

32. Shirazikhah M, Mirabzadeh A, Sajadi H, Joghataei MT, Biglarian A, Mousavi $\mathrm{T}$, et al. National survey of availability of physical rehabilitation services in Iran: A mixed methods study. Electron Physic. 2017;9(11):5778.

33. Ahmadzadeh N, Azar FEF, Baradaran HR, Roghani RS, Shirazikhah M, Joghataei MT, et al. Availability of outpatient rehabilitation facilities in 2015 in Iran: A nationwide study. Med J Islam Repub Iran. 2018;32:123 
Appendix 1. Results of selected articles were made available to the specialists

\begin{tabular}{|c|c|c|c|c|}
\hline Cods extracted from the study & Study & Goals of the study & $\begin{array}{c}\text { Author \& } \\
\text { year }\end{array}$ & $\mathrm{n}$ \\
\hline $\begin{array}{l}\text { 1-clients' attitudes about personal and practice characteristics of existing providers } \\
\text { including age, sex 2- Location and type of Facility or religious affiliation of provid- } \\
\text { er or facility, as well as provider attitudes about acceptable personal characteristics } \\
\text { of clients, including ethnicity and patient payment Source. 3-overall satisfaction }\end{array}$ & survey & $\begin{array}{c}\text { Measuring the Acceptability to } \\
\text { Cardiovascular services in Rural } \\
\text { and Remote Australia via Applied } \\
\text { Geographical Spatial Technology } \\
\text { (GIS) project }\end{array}$ & $\begin{array}{c}\text { (Van Gaans, } \\
\text { 2013) }\end{array}$ & 1 \\
\hline $\begin{array}{l}\text { about sex of personal and practice } \\
\text { satisfaction of skill of personnel treatment } \\
\text { cleanliness of facility cultural and religious } \\
\text { satisfaction of personal } \\
\text { overall satisfaction }\end{array}$ & Case study & $\begin{array}{l}\text { evaluate acceptability to primary } \\
\text { healthcare }\end{array}$ & $\begin{array}{l}\text { (Shrestha, } \\
\text { 2010) }\end{array}$ & 2 \\
\hline $\begin{array}{l}\text { satisfaction of skill of personnel treatment } \\
\text { overall satisfaction }\end{array}$ & review & $\begin{array}{l}\text { Design framework for analysis and } \\
\text { action to explore and improve } \\
\text { acceptability to health care in } \\
\text { resource-poor countries }\end{array}$ & $\begin{array}{l}\text { (Obrist et } \\
\text { al., 2007) }\end{array}$ & 3 \\
\hline $\begin{array}{c}\text { cultural and religious in prefer chose of facility } \\
\text { overall satisfaction }\end{array}$ & review & $\begin{array}{l}\text { acceptability to medicines and } \\
\text { principles }\end{array}$ & $\begin{array}{l}\text { (Pogge, } \\
2008)\end{array}$ & 4 \\
\hline $\begin{array}{c}\text { satisfaction of personal } \\
\text { overall satisfaction }\end{array}$ & review & $\begin{array}{l}\text { The use of numerical indicators to } \\
\text { measure economic and social } \\
\text { conditions and progress toward } \\
\text { adopted goals has become accepted } \\
\text { practice by many national and } \\
\text { international agencies }\end{array}$ & $\begin{array}{l}\text { (May et al., } \\
\text { 2000) }\end{array}$ & 5 \\
\hline
\end{tabular}

Appendix 2. Rehabilitation Services Acceptability Questionnaire

\begin{tabular}{llllll}
\hline Questions & Never & Rarely & Sometimes & Often & Always \\
\hline
\end{tabular}

To what degree would you rate your satisfaction to the explanations given by the

health care provider while doing his rehabilitation services?

In your opinion, how would you rate you're your satisfaction with the type of relation-

ships provided by your rehabilitation service provider?

How well did your service provider spend his time in listening to your problems and

other issues while rendering his services?

How would you rate the professional skills and competencies of you rehabilitation

service provider?

How would you rate the attention given by your health care provider and the respect for your privacy in rendering health services?

How respectful and attentive were the health service providers when rendering their services?

As a whole, how would you rate the services being rendered to you?

How would you rate your involvement in the planning of rehabilitation services?

How would you rate the services of the health provider in terms of gender?

How would you rate the environmental condition of the health service facility in

terms of (lighting, noise, ventilation, cleanliness and suitable temperature)?

How would you rate the respect for your right in receiving rehabilitation services?

How would you rate the use of harsh and unscrupulous methods of treatment as the reason for your discontinuance of rehabilitation services?

How would you rate inattention or neglect of the health service provider to your religious beliefs as a reason for discontinuance of rehabilitation services?

How would you rate carelessness and the lack of time spent by the health care provider as a reason for discontinuance of your rehabilitation services?

How would you rate the inattention of the health service provider to culture, customs and traditions and ethnicity as a reason for the discontinuance of your rehabilitation services?

How would you rate disclosure of your information to others as the reason for your discontinuance of your rehabilitation services?

How would you rate the reason of your refusal to receive further rehabilitation services because your health care provider believed that your condition would not improve?

How would you rate the lack of attention by health service provider to your condition as the reason for discontinuing your health services?

How would you rate your feeling that your physical and Psychological problems is of no importance to the health care provider?

How would you rate your experience of rejection from the Rehabilitation Services

Center as the reason for discontinuance of the rehabilitation services?

How would you rate the absence of private room for examination and for performing rehabilitation services as the reason for your discontinuance of the rehabilitation services? 